\title{
NEURAL SYMBOLIC ARABIC PARAPHRASING WITH AUTOMATIC EVALUATION
}

\author{
Fatima Al-Raisi, Abdelwahab Bourai and Weijian Lin \\ Language Technologies Institute, School of Computer Science, \\ Carnegie Mellon University, Pittsburgh, PA, USA
}

\begin{abstract}
We present symbolic and neural approaches for Arabic paraphrasing that yield high paraphrasing accuracy. This is the first work on sentence level paraphrase generation for Arabic and the first using neural models to generate paraphrased sentences for Arabic. We present and compare several methods for para- phrasing and obtaining monolingual parallel data. We share a large coverage phrase dictionary for Arabic and contribute a large parallel monolingual corpus that can be used in developing new seq-to-seq models for paraphrasing. This is the first large monolingual corpus of Arabic. We also present first results in Arabic paraphrasing using seq-to-seq neural methods. Additionally, we propose a novel automatic evaluation metric for paraphrasing that correlates highly with human judgement.
\end{abstract}

\section{KEYWORDS}

Natural Language Processing, Paraphrasing, Sequence-to-Sequence Models, Neural Networks, Automatic Evaluation, Evaluation Metric, Data Resource

\section{INTRODUCTION}

Paraphrasing and paraphrase detection are two important problems in natural language processing. Paraphrasing-based applications include text simplification and text generation form structured knowledge [19]. Other paraphrastic models include machine translation and sentence summarization $[14,24,6]$. Paraphrases are useful not only in generation tasks but also in analysis tasks such as information retrieval and question answering [25, 20,9].

We present and compare two different approaches for sentence paraphrasing in Arabic: a phrasebased method and a neural method. To our knowledge, this is the first work on sentence paraphrasing for modern standard Arabic.

We also present a novel approach for obtaining parallel monolingual data and use the acquired data to train our neural sequence-to-sequence model. As a by-product of this work, we contribute a large parallel monolingual corpus for Arabic containing two million sentence pairs which can be used to develop new seq-to-seq models for paraphrasing. We also build a phrase database for Arabic containing over $88 \mathrm{~K}$ phrase pairs of various lengths. Another contribution of our work is

Dhinaharan Nagamalai et al. (Eds) : NATL, CSEA, DMDBS, Fuzzy, ITCON, NSEC, COMIT - 2018 pp. 01-13, 2018. @ CS \& IT-CSCP 2018

DOI : $10.5121 /$ csit.2018.80601 
Computer Science \& Information Technology (CS \& IT)

devising and testing a new evaluation metric for paraphrasing. We present encouraging initial results using this metric in this paper. The remainder of this paper is structured as follows: we contextualize our work within paraphrasing research in Section 2, we present the phrase-based and neural approaches for paraphrasing sentences and building phrase dictionaries in sections 3 and 4. We present details and discuss experiments on the evaluation metric in Section 6 . We conclude with plans for future extensions of the work.

\section{RELATED WORK}

The paraphrase database project PPDB has paraphrase resources for multiple languages [3], including Arabic. The paraphrases are obtained using parallel bilingual corpora by applying the pivot method where one language is used as a bridge or intermediate meaning representation [3]. Paraphrases from dialectal Arabic to standard Arabic have been used in [21] to improve ArabicEnglish statistical machine translation. Turker assisted paraphrasing has been used in [8] to improve English-Arabic MT. A comparison between various paraphrase acquisition techniques on sentential paraphrasing is given in [5] but does not include experiments on Arabic sentential paraphrasing.

\section{EXTRACTING PARAPHRASES FROM BILINGUAL DATA}

Our first approach to Arabic paraphrasing is the pivot method proposed by Bannard and CallisonBurch [3]. A key benefit is that it is language-agnostic and is based on the idea that any two source strings $e_{1}$ and $e_{2}$ that both translate to a reference string $f_{1}$ have similar meaning. Bannard and Callison-Burch used English as the reference string f, but in our study we will instead pivot into English to obtain paraphrase pairs [3]. We obtain the final paraphrase probability by marginalizing over the English translation probabilities with e and Arabic phrases $\mathrm{a}_{1}$ and $\mathrm{a}_{2}$. A mathematical formulation of the approach can be found in Equation 1.

$$
p\left(a_{2} \mid a_{1}\right)=\sum_{e} p\left(a_{2} \mid e\right) p\left(e \mid a_{1}\right)
$$

In order to extract paraphrases, we first obtained a parallel bilingual corpus through English and Arabic versions of the EUROPARL dataset [13]. We pruned the corpus to only contain sentences with less than 80 words and tokenized using the StanfordNLP Arabic Tokenizer [15]. This was achieved in the data preprocessing step by computing the sentence length and excluding sentences with more than 80 tokens. This gave us a final corpus size of 241,902 sentences.

Additionally, to calculate conditional probabilities for our paraphrase equation, we need alignment. Thus we ran GIZA++ [18], the well-known alignment tool widely used in MT, to obtain these alignments [18]. We chose GIZA++ for its previous success with machine translation involving Arabic [1]. Once we have a database of paraphrase mappings, we can then substitute phrases with their corresponding paraphrases by selecting the phrase with the highest probability. This substitution approach was used by Bannard and Callison-Burch in their study as well [3] The way we extract the paraphrase is summarized in Equation 2. An example of this process can be seen in Figure 1

$$
\hat{a_{2}}=\operatorname{argmax}_{a_{2} \neq a_{1}} p\left(a_{2} \mid a_{1}\right)
$$




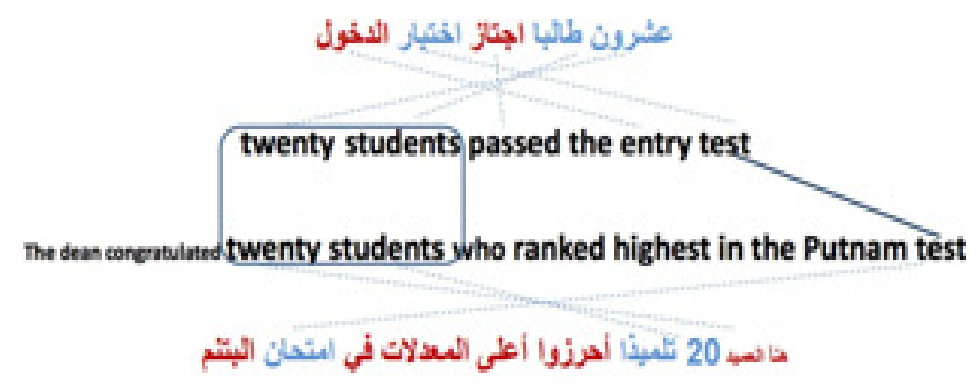

Figure 1: An example paraphrased sentence produced using the pivot method.

\subsection{Improving Coverage of Phrase Database}

In our initial experiments, we noticed that generated phrase pairs did not necessarily match in some grammatical features such as definiteness and number. We post-processed the phrase dictionary to add entries with other variants of the phenomenon for completion. For example, for a word pair that appears in the phrase table where one word is definite and the other is not, we add two entries where both are definite and both are indefinite. We did not adjust the scores to reflect this but ordered the entries according to observed frequency of the word/phrase. For definiteness, we limited the addition to the clear definite marker "al-" in Arabic. We applied the same for simple cases of number matching where the morphology is concatenative or easily processed. We note that this modifications did not include all possible mismatches since they were based on simple heuristics. However, this may have contributed to better grammaticality as discussed in Section 5. We also noticed cases like the following in the generated phrase table:

x III y III score

x III z III score

We included, for improved coverage, the following entry:

y III z III score

Again, this was limited in scope since we relied on simple string match to identify such entries.

\subsection{Phrase-substitution}

We randomly sampled 100 sentences from the datasets we have [23, 12] and performed phrase substitution. Figure 2 shows a sample of paraphrased sentences acquired using phrase substitution. We note that in the last example the output sentence differs from the original in only one word (last word) but the meaning is entirely altered. We discuss results and experiments on the quality of paraphrased sentences in Section 5. 


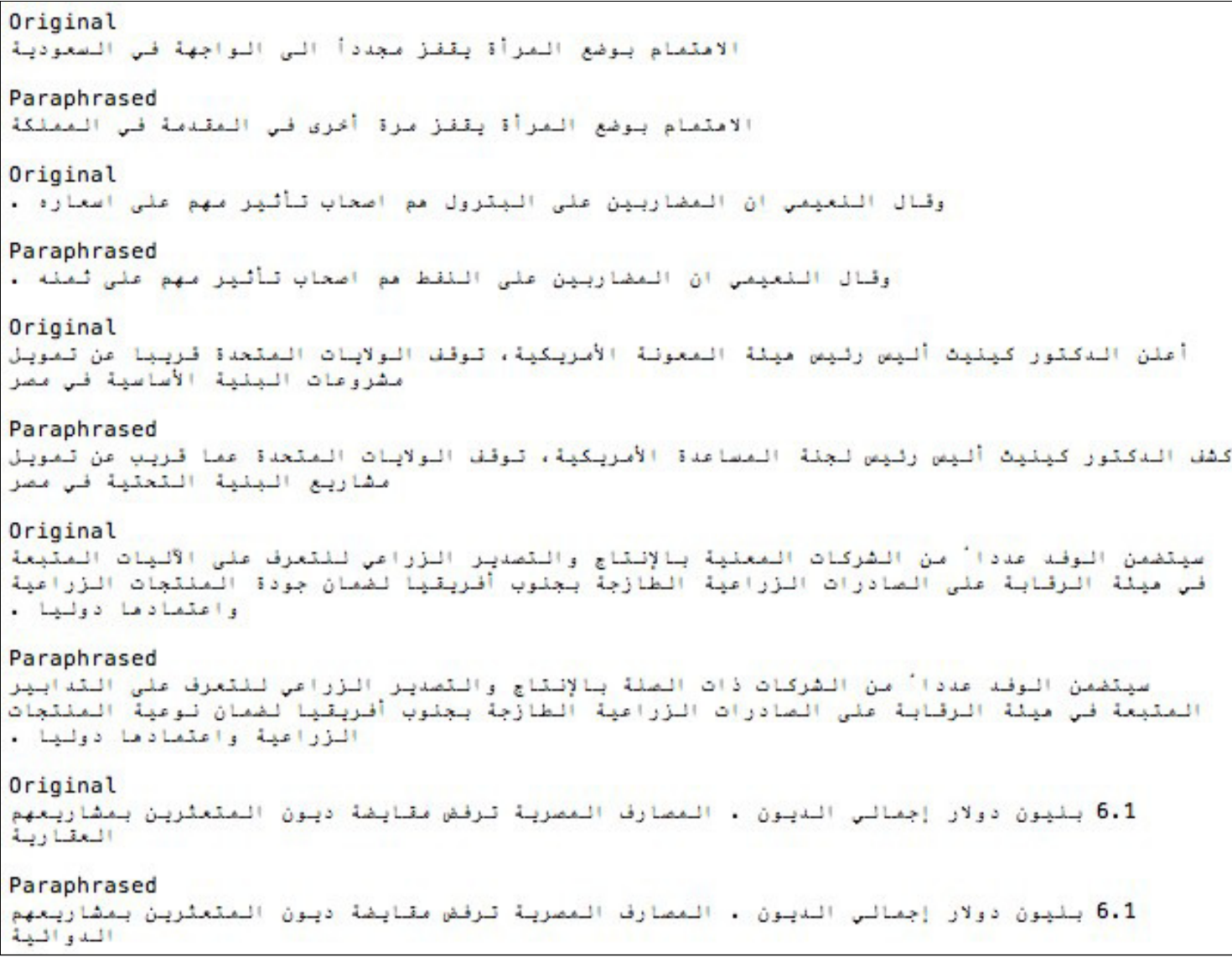

Figure 2: Sample output produced using phrase substitution

\section{Monolingual Parallel data For SeQ-To-SeQ PARAPHRASING}

We need parallel monolingual data to train our sequence-to-sequence paraphrasing model. To address the lack of parallel monolingual data for Arabic, we propose a novel method for generating Arabic parallel language sentences using two other language pairs as resource. The idea is to use paired sentences data from two other languages, translate them into Arabic correspondingly, then use them as the training data for sequence-to-sequence machine translation model to train a translator to generate Arabic to Arabic paraphrases.

The first advantage of this approach is its scalability. After preparing enough training data for the paraphrase model, the generation step for Arabic paraphrases is easily scalable. The second advantage is that the seq-to-seq paraphraser model may contain valuable insight for building Arabic paraphrases database at words and phrases level, since state of the art neural machine translation techniques are capable of capturing word and phrase level similarity by projecting word embeddings into vector space.

We used europarl-v7 fr-en [13] data which contains two million sentence pairs, and then used Google translate API to generate French-Arabic and English-Arabic sentence pairs 
correspondingly. Then we paired the output to construct parallel monolingual data and used it as training data for a Bi-LSTM with embedding size and hidden size set to be 512 and attention size set to be 128. The bidirectional LSMT model is chosen to create a stronger representation that takes into account patterns found in the right-to-left Arabic sentence direction as well as features and patterns present towards the end of the sentence which are attenuated in right-to-left unidirectional representation.

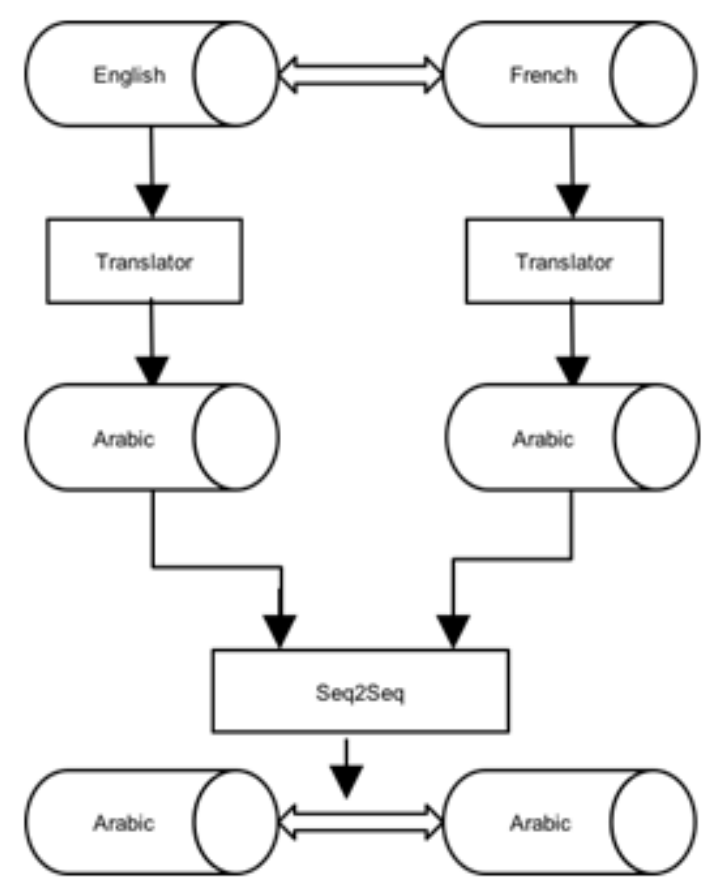

Figure 3: Overview of the process completed to obtain two parallel Arabic corpora generated from different source languages

The grammaticality of the Arabic output sentences were independently evaluated by two speakers of Arabic and found to be both grammatical and semantically coherent. The process is demonstrated in Figure 3. Training the Bi-LSTM took about 7 days on this dataset and we obtained a corpus of monolingual Arabic containing two million parallel sentences.

\section{RESULTS}

We report results on the bilingual pivot and sequence-to-sequence approaches detailed above.

\subsection{Phrase-based Method}

Using the pivot method, we obtained over $88 \mathrm{~K}$ phrase pairs. We report a few results. First, Figure 4 shows the length distribution for phrases in the database.

We obtained human evaluations from two native speakers on the grammaticality and meaning preservation aspects of the paraphrased sentences using a sample of 200 sentence pairs. We note that this sample is more than two times larger than samples used in similar evaluations in 
related work [17]. For each criterion, we asked the annotator to judge the quality of the output on a scale from 1 to 5 . We chose this scale to capture variation in the level of grammaticality (since there are minor and more serious grammatical mistakes) and in the extent to which the paraphrased sentence preserved the meaning of the original sentence. The agreement between annotators calculated in terms of IntraClass Correlation, preferred for ordinal data, is summarized in Table 1. It was not expected to find higher agreement on meaning preservation since it is more subjective than grammaticality. It is possible that phrases substituted were of similar meaning yet possibly resulted in unusual sentence structure which made the meaning preservation judgement straightforward while grammaticality harder to judge. We analyzed changes made to reference sentences that received the highest score in meaning preservation after paraphrase substitution $($ score $=5)$. A change is a word replacement or deletion. Table 2 summarizes these changes. When $\mathrm{n}$ consecutive words are replaced by $\mathrm{n}$ or more words, we consider those to be $\mathrm{n}$ changes rather than 1 phrasal change. Table 3 summarizes the evaluation of paraphrased sentences obtained using phrase substitution.

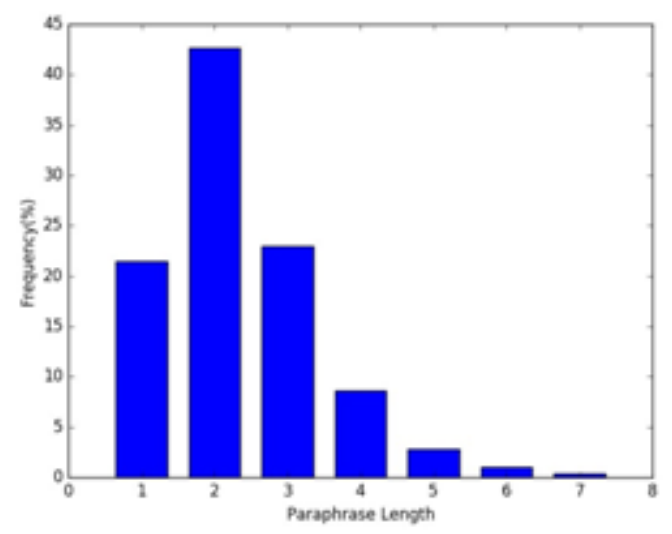

Figure 4: Distribution of phrase lengths in our paraphrases obtained through pivoting

Table 1: Inter-annotator agreement

\begin{tabular}{|c|c|c|}
\cline { 2 - 3 } \multicolumn{1}{c|}{} & ICC ( single) & ICC (average) \\
\hline Grammaticality & 0.375 & 0.545 \\
\hline Meaning & 0.707 & 0.547 \\
\hline
\end{tabular}

\subsection{Neural Method}

The corpus of sentence pairs produced was large (2M). By the time the output was produced, our human evaluation resources were exhausted, so we qualitatively evaluate it on a subset consisting of 200 sentence pairs. The monolingual parallel sentences obtained were found to be grammatical or near grammatical with minor mistakes but were found to be highly similar in meaning. Also, they were sufficiently different in surface form which encouraged their use as training data for the a seq-to-seq paraphrasing model. Initially, we were concerned of the possibility that the en $\rightarrow$ ar and $\mathrm{fr} \rightarrow$ ar translation tools we used were trained on the same Arabic data with similar model architecture and parameters, in which case the output would not be diverse enough to be useful as input to the paraphrasing system. We were also concerned of the possibility that the French source was translated to English and then to Arabic. However, judging by the diversity in the output of these models, using the resultant parallel monolingual corpus to 
train the neural paraphrasing model is justified. We compare the surface diversity in the output of the fr-ar and en-ar MT models by computing word and phrase overlap and find enough surface variation to train the model adequately.

Table 2: Number of changes in paraphrased sentences with high meaning preservation score

\begin{tabular}{|c|c|c|c|c|c|}
\hline No.of Changes & 5 & 4 & 3 & 2 & 1 \\
\hline Frequency (Sent) & 3 & 4 & 5 & 16 & 26 \\
\hline
\end{tabular}

Table 3: Evaluation of paraphrased sentences (averaged and rounded from two annotator ratings)

\begin{tabular}{|c|c|c|c|}
\hline Scale & Grammaticality $\%$ & Meaning $\%$ & Both $\%$ \\
\hline 5 & 62 & 62 & 50 \\
\hline 4 & 21 & 16 & 7 \\
\hline 3 & 10 & 9 & 3 \\
\hline 2 & 7 & 12 & 4 \\
\hline 1 & 0 & 1 & 0 \\
\hline
\end{tabular}

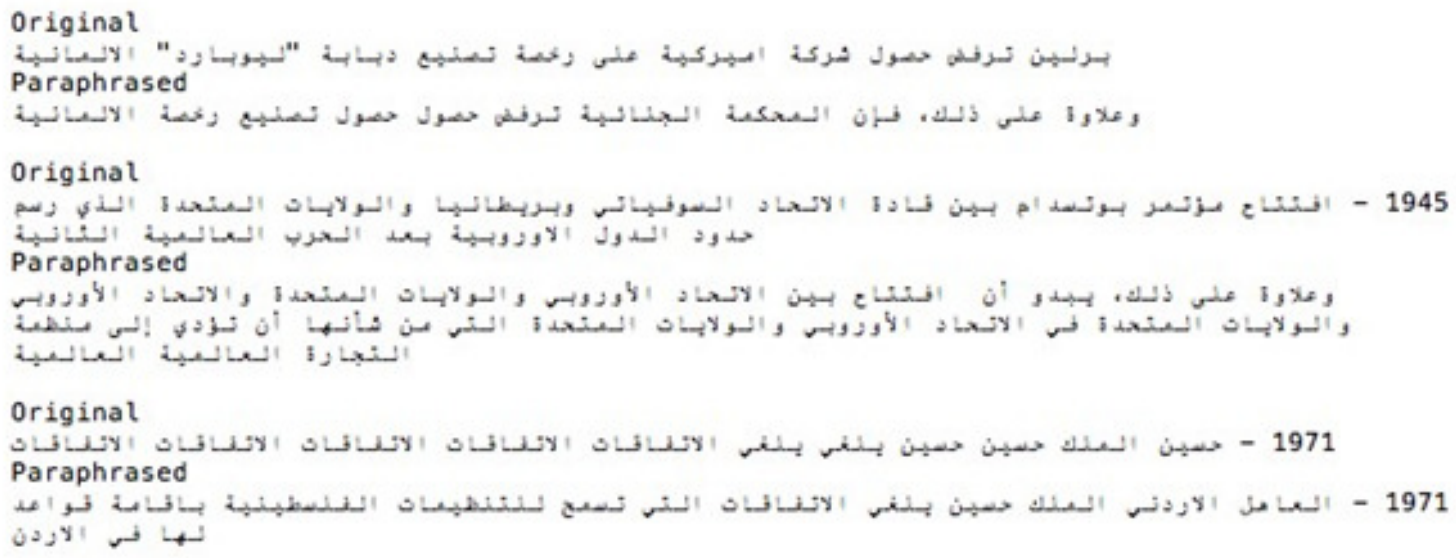

Figure 5: Sample output from neural parpahrasing model

The paraphrased output from the neural system was far from grammatical and in some cases the meaning was incomplete due to early sentence truncation, especially for long sentences. Better output was observed for shorter sentences which conforms with the pattern typically seen in neural seq-to-seq models. However, we make the following observations about the output:

- The model learns what phrases to use at the beginning of the sentence. It uses things like "Moreover" and "As you know," (translated from Arabic), exactly at the beginning of the sentence.

- The model seem to learn and include central parts of the sentence in the output such as the subject or the location of the event.

- The model learns correspondences between the main parts of the sentence; e.g., the byline vs. remainder of the sentence and quoted text vs. part before the quotation.

- The model often fails in producing output with the correct word order.

- As observed with neural language models, it tends to repeat words. 
Figure 5 shows a sample output from the neural model.

\section{AN Automatic Evaluation Metric}

Since human evaluation is time-consuming, subjective and not always available, we propose an automatic evaluation metric for paraphrasing. We propose criteria for judging paraphrase quality and operationalize those criteria using well-defined functions. A good paraphrase has the following two properties:

1. maintains the meaning of the original text, yet

2. expresses the same meaning using different surface realizations.

To evaluate the semantic similarity and surface variation in a paraphrase we employ well-defined metrics discussed next.

\subsection{Semantic Similarity}

Several methods exist for capturing the semantic similarity of text $[11,7,2]$. One simple approach uses the distributional properties of words in the sentence and embeds a sentence by averaging the embedding vectors of its words. We choose this method for its simplicity and efficiency. The sentence vector is thus given by:

$$
w_{x}=\frac{1}{|x|} \sum_{1}^{n} w_{x_{i}}
$$

where $n$ is the number of words in the sentence. Although this method is simple and does not consider word-order or syntactic structure, it performs surprisingly well when compared to more advanced neural- based methods designed to capture sentential semantics [22]. It also correlates highly with human judgement [22]. Also, being agnostic to word order is actually a desired property in the paraphrase case since valid paraphrases may only differ in the order of the words or the construction used. For example, in Arabic the SVO word order can almost always be changed to VSO without changing the meaning of the sentence (except for emphasis) and without introducing any other particle. Another example is English active voice and the corresponding passive voice sentence ( + by Subj) of the original sentence.

To compute the semantic similarity between two sentences, the original sentence and the paraphrase, the cosine similarity between the two sentence vectors is computed. In our experiments, we use word embeddings with 300 dimensions trained on Arabic text from [4]. Cosine similarity is chosen for its efficient and parallelizable computation. An evaluation metric should be computed efficiently for it to be useful in evaluating output and comparing several systems performance in real-time. Note that the purpose of the metric is not to capture the most accurate distance between the vectors in space per se but to efficiently and reasonably estimate the similarity between the sentences in a principled way which cosine similarity suffices for. In our generic framework, this metric could be replaced with any other semantic similarity metric of choice. 


\subsection{Surface Variation}

To capture surface variation we first map each sentence into a common vocabulary space and compute the hamming distance between the sentence vectors in that space. This also limits sentence length bias where short sentences will naturally have less surface overlap. In our experiments, we map sentences into a vocabulary space of 610977 words [4]. We present experiments and results in section 6.4.

\subsection{Combining Criteria for Meaning and Form}

Minimal change in surface form can result in maximal preservation of original meaning. However it will score low on surface variation. Similarly, if surface form is significantly changed, we may risk altering the meaning of the original sentence. Since semantic similarity and surface variation are two competing criteria, we combine them using (balanced) harmonic mean. The final score of the paraphrase is given by:

$$
s=2 \frac{\text { SemanticSimilarity } \cdot \text { LexicalDistance }}{\text { SemanticSimilarity }+ \text { LexicalDistance }}
$$

\subsection{Results}

We evaluate a set of 198 Arabic sentence pairs sampled from newswire data [12] as follows. These include headlines on similar topics and for each headline one or two sentences detailing the event in the headline or reiterating it. Pairs including the headline and the following sentence were reasonable paraphrase pairs whereas the other pairs varied in paraphrasing potential from moderate (some overlap in meaning) to poor (unrelated, contradicting or little overlap). We created 576 sentence pairs from the dataset but obtained annotations for only 198 of them. This evaluation of the metric was conducted before obtaining paraphrasing results from our phrasebased and neural models. Therefore, we created sentence and paraphrase pairs following this approach. Since sentences were obtained from newswire data, we assumed they are grammatical and did not obtain grammatical judgement from annotators. On paraphrastic quality, human evaluations were obtained from three annotators who are native speakers of Arabic. Each annotator was asked to judge the quality of the paraphrase, on whether it preserved meaning and was expressed differently, on a ordinal scale from 1 to 5 where 1 indicates poor quality. We used $\mathrm{R}$ to measure interannotator (absolute) agreement using IntraClass Correlation (ICC) and agreement was measured at 0.714 ICC which is considered "very good." The biserial correlation between the binarized human evaluations and the evaluation metric scores was 0.813 .

\subsection{Analysis}

Observing high correlation between human evaluation and the proposed evaluation metric, we examined the dataset to see if results were biased by sampling or data peculiarities. For sentence pairs including the headline and the following sentence, both human and evaluation metric scores were high. For most of the other sentence pairs, the paraphrase was judged as weak or poor. In both of these cases, the judgement was "easy" and straightforward and this perhaps lead to the surprisingly good results. Perhaps sentence pairs with finer and more subtle semantic phenomena such as polysemy and synonymy would have been harder to score accurately by the metric. We need to conduct more experiments to verify this. 
We also explored the effect of the embedding dimension on the correlation between human evaluation and the metric we proposed. We experimented with lower dimensions: 50, 100, 150, 200, 250 and obtained slightly lower values of biserial correlation as we decreased the embedding dimension. With 50 dimensions, the absolute difference between the previous biserial correlation value and the new one was 0.03 . It is worth mentioning that when only using overlap as a measure of semantic similarity, the correlation between human judgement and the evaluation metric is 0.47 . We clearly gain by using word embeddings but even something as simple as word overlap can capture semantic distance to some extent and explain a good amount of variation in human judgement.

We initially set out to compute the surface distance between two sentences using a measure that only depends on the two sentences as input; such as token/character overlap or minimum edit distance. However, since this measure can have a length bias we use the more robust hamming distance which is computed in a canonical space.

Using a common canonical space for doing the various computations is preferred as these spaces comprise a reference against which candidates are evaluated. When using the metric to evaluate outputs from different systems, the reference can be decided at test time to avoid "gaming" the metric. It is desirable to have a metric that does not require a reference sentence when evaluating a candidate paraphrase since 1. results and rankings can be sensitive to the choice of the reference sentence which is often subjective and 2. the notion of a "reference paraphrase" is problematic here since paraphrasing is essentially based on divergence from a given surface form while preserving the underlying meaning and hence is loosely constrained. However, we do recognize the problem with not having a reference for an evaluation metric: it can make it susceptible to "gaming" by players competing to optimize the metric score. Therefore, we propose to decide the vocabulary space in which surface distance function is computed and the semantics space in which semantic similarity is computed at test time.

Also, since this metric combines competing objectives, the parameter controlling the relative strength of each component can also be decided at test time to improve the metric robustness. While it has been shown that it is possible to find a Pareto optimal hypothesis that aims to jointly optimize several different objectives [10], we argue that those objectives are not exactly competing or orthogonal. They may be weakly correlated but they are still positively correlated since they compare surface distance against the same reference (as in BLUE and TER), which is not the case in our proposed metric setting.

\section{CONCLUSION}

We presented and compared two different approaches for sentence paraphrasing in Arabic. The phrase-based approach yielded very good results when used to create paraphrase sentences. The neural method output is still far from practical applicability but the model has learned interesting linguistic constructs like phrases used for sentence opening. We also presented a novel approach for obtaining parallel monolingual data and contributed a dataset of two million parallel sentence pairs in Arabic using this approach. We applied the pivoting method to construct a large coverage paraphrase database for Arabic that includes over $88 \mathrm{~K}$ phrase pairs. When used to create new sentences, the paraphrase dictionary gave very good results on grammaticality and meaning preservation. We proposed a new automatic evaluation metric for paraphrasing that 
does not require the use of a reference sentence while evaluating candidate hypotheses. We showed encouraging preliminary results in terms of correlation with human judgement.

\section{FUTURE WORK}

We plan to explore other options for obtaining monolingual parallel data. One possible approach is to retrieve headlines of news articles from different agencies covering the same event. We expect headlines describing the same event to have some degree of semantic similarity yet different surface realizations.

The sequence-to-sequence model required a relatively long time to run which limited the testing of other architectures and model parameters. We plan to conduct more experiments on different architectures and compare results. More specifically, whether we get better results with a unidirectional model since sequences from the same language will tend to have similar word order. We also intend to incorporate the concept of "coverage" [16] to address issues with fluency of the neural model output.

We obtained encouraging results from the evaluation metric experiments but we need to verify its usefulness in settings with subtle semantic phenomena such as polysemy and synonymy. We also plan to use it at word subunits such as morphemes and even character level especially for surface distance comparison in morphology rich languages like Arabic.

\section{REFERENCES}

[1] Shady Abdel Ghaffar and Mohamed Fakhr. English to arabic statistical machine translation system improvements using preprocessing and arabic morphology analysis. Recent Researches in Mathematical Methods in Electrical Engineering and Computer Science, pages 50-54, 122011.

[2] Eneko Agirre, Enrique Alfonseca, Keith Hall, Jana Kravalova, Marius Passca, and Aitor Soroa. A study on similarity and relatedness using distributional and wordnet-based approaches. In Proceedings of Human Language Technologies: The 2009 Annual Conference of the North American Chapter of the Association for Computational Linguistics, NAACL '09, pages 19-27, Stroudsburg, PA, USA, 2009. Association for Computational Linguistics.

[3] Colin Bannard and Chris Callison-Burch. Paraphrasing with bilingual parallel corpora. In Proceedings of the 43rd Annual Meeting on Association for Computational Linguistics, ACL'05, pages 597-604, Stroudsburg, PA, USA, 2005. Association for Computational Linguistics.

[4] Piotr Bojanowski, Edouard Grave, Armand Joulin, and Tomas Mikolov. Enriching word vectors with subword information. arXiv preprint arXiv:1607.04606, 2016.

[5] Houda Bouamor, Aurélien Max, and Anne Vilnat. Comparison of paraphrase acquisition techniques on sentential paraphrases. In Advances in Natural Language Processing: 7th International Conference on NLP, IceTAL 2010, Reykjavik, Iceland, August 16-18, 2010, pages 67-78, Berlin, Heidelberg, 2010. Springer Berlin Heidelberg.

[6] Chris Callison-Burch, Philipp Koehn, and Miles Osborne. Improved statistical machine translation using paraphrases. In Proceedings of the Main Conference on Human Language Technology Conference of the North American Chapter of the Association of Computational Linguistics, HLTNAACL '06, pages 17-24, Stroudsburg, PA, USA, 2006. Association for Computational Linguistics. 
[7] Courtney Corley and Rada Mihalcea. Measuring the semantic similarity of texts. In Proceedings of the ACL Workshop on Empirical Modeling of Semantic Equivalence and Entailment, EMSEE '05, pages 13-18, Stroudsburg, PA, USA, 2005. Association for Computational Linguistics.

[8] Michael Denkowski, Hassan Al-Haj, and Alon Lavie. Turker-assisted paraphrasing for english-arabic machine translation. https://www.cs.cmu.edu/ mdenkows/pdf/paraphrasemturk- 2010.pdf, 2010.

[9] Peter Wallis Dept and Peter Wallis. Information retrieval based on paraphrase. In In Proceedings of PACLING Conference, 1993.

[10] Kevin Duh, Katsuhito Sudoh, Xianchao Wu, Hajime Tsukada, and Masaaki Nagata. Learning to translate with multiple objectives. In Proceedings of the 50th Annual Meeting of the Association for Computational Linguistics: Long Papers - Volume 1, ACL '12, pages 1-10, Stroudsburg, PA, USA, 2012. Association for Computational Linguistics.

[11] Rafael Ferreira, Rafael Dueire Lins, Fred Freitas, Steven J. Simske, and Marcelo Riss. A new sentence similarity assessment measure based on a three-layer sentence representation. In Proceedings of the 2014 ACM Symposium on Document Engineering, DocEng '14, pages 25-34, New York, NY, USA, 2014. ACM.

[12] David Graff and Kevin Walker. Arabic newswire part 1, 2001.

[13] Philipp Koehn. Europarl: A parallel corpus for statistical machine translation. http://www.statmt.org/europarl/, 2005.

[14] Philipp Koehn, Franz Josef Och, and Daniel Marcu. Statistical phrase-based translation. In Proceedings of the 2003 Conference of the North American Chapter of the Association for Computational Linguistics on Human Language Technology - Volume 1, NAACL '03, pages 48-54, Stroudsburg, PA, USA, 2003. Association for Computational Linguistics.

[15] Christopher D. Manning, Mihai Surdeanu, John Bauer, Jenny Finkel, Steven J. Bethard, and David McClosky. The Stanford CoreNLP natural language processing toolkit. In Association for Computational Linguistics (ACL) System Demonstrations, pages 55-60, 2014.

[16] Haitao Mi, Baskaran Sankaran, Zhiguo Wang, and Abe Ittycheriah. A coverage embedding model for neural machine translation. CoRR, abs/1605.03148, 2016.

[17] Masahiro Mizukami, Graham Neubig, Sakriani Sakti, Tomoki Toda, and Satoshi Nakamura. Building a free, general-domain paraphrase database for japanese. In The 17th Oriental COCOSDA Conference, Phuket, Thailand, September 2014.

[18] Franz Josef Och and Hermann Ney. A systematic comparison of various statistical alignment models. Computational Linguistics, 29(1):19-51, 2003.

[19] Ellie Pavlick and Chris Callison-Burch. Simple PPDB: A paraphrase database for simplification. In Proceedings of the 54th Annual Meeting of the Association for Computational Linguistics, ACL 2016, August 7-12, 2016, Berlin, Germany, Volume 2: Short Papers. The Association for Computer Linguistics, 2016.

[20] Fabio Rinaldi, James Dowdall, Kaarel Kaljurand, Michael Hess, and Diego Mollá. Exploiting paraphrases in a question answering system. In Proceedings of the Second International Workshop on Paraphrasing - Volume 16, PARAPHRASE '03, pages 25-32, Stroudsburg, PA, USA, 2003. Association for Computational Linguistics. 
[21] Wael Salloum and Nizar Habash. Dialectal to standard arabic paraphrasing to improve arabic english statistical machine translation. In Proceedings of the First Workshop on Algorithms and Resources for Modelling of Dialects and Language Varieties, DIALECTS '11, pages 10-21, Stroudsburg, PA, USA, 2011. Association for Computational Linguistics.

[22] John Wieting, Mohit Bansal, Kevin Gimpel, and Karen Livescu. Towards universal paraphrastic sentence embeddings. CoRR, abs/1511.08198, 2015.

[23] Ma Xiaoyi, Dalal Zakhary, and Moussa Bamba. Arabic news translation text part 1 ldc2004t17. https://catalog.ldc.upenn.edu/LDC2004T17, 2004.

[24] Liang Zhou, Chin-Yew Lin, Dragos Stefan Munteanu, and Eduard Hovy. Paraeval: Using paraphrases to evaluate summaries automatically. In Proceedings of the Main Conference on Human Language Technology Conference of the North American Chapter of the Association of Computational Linguistics, HLT-NAACL '06, pages 447-454, Stroudsburg, PA, USA, 2006. Association for Computational Linguistics.

[25] Ingrid Zukerman, Bhavani Raskutti, and Yingying Wen. Experiments in query paraphrasing for information retrieval. In AI 2002: Advances in Artificial Intelligence: 15th Australian Joint Conference on Artificial Intelligence Canberra, Australia, December 2-6, 2002 Proceedings, pages 24-35, Berlin, Heidelberg, 2002. Springer Berlin Heidelberg 